\title{
APROVECHAMIENTO DE PIGMENTOS ANTOCIÁNICOS DEL MAÍZ MORADO (Zea mays l) EN LA ELABORACIÓN DE UNA BEBIDA ALCOHÓLICA TIPO VINO
}

\author{
THE USE OF ANTHOCYANIN PIGMENTS CORN PURPLE (Zea \\ mays l) IN THE MANUFACTURE OF AN ALCOHOLIC \\ BEVERAGE WINE TYPE
}

\author{
Samuel Román Cerro Ruiz
}

\begin{abstract}
RESUMEN
El presente trabajo de investigación busca determinar la posibilidad de transferir la riqueza que poseen los granos y especialmente las corontas de maíz morado (Zea mays L), al transformarlo en una bebida alcohólica tipo vino.

Se trabajó con cuatro muestras ( $M-1, M-2, M-3$ y $M-4$ ) que tuvieron la siguiente composición: $50 \%$ de granos malteados, $10 \%$ de granos no malteados y $40 \%$ de corontas. Una vez molidos, fueron puestos a calentar durante 1 hora, a una temperatura que va de $75-85^{\circ} \mathrm{C}$ y en cuatro niveles de agua $(M-1=6, O \mathrm{~L}, \mathrm{M}-2=8,0 \mathrm{~L}, \mathrm{M}-3=10,0 \mathrm{~L}$ y $\mathrm{M}-4=12, \mathrm{OL})$. Los mostos cocidos incrementaron en 3 y hasta 5 veces el valor inicial de las antocianinas mostradas por las corontas secas; respecto a granos de maíz morado, los incrementos en antocianinas fueron entre 7 y casi 11 veces más debido a los procesos de extracción por lixiviación en caliente.
\end{abstract}

Para favorecer el proceso fermentativo de los cuatro mostos, se agregó jarabe acidulado de sacarosa, hasta elevar sus sólidos solubles de 5 a $22.5^{\circ}$ Brix, así como también aumentar en $3 \%$ el volumen de un fermento de maíz blanco como pie de cuba.

Se logró cuatro vinos de maíz morado que se conservaron y decantaron cuatro veces, durante 90 días y contenían una graduación alcohólica entre $9^{\circ}$ y $10^{\circ} \mathrm{GL}, \mathrm{pH}$ entre 3.1 y 3.3, así como contenidos en antocianinas, entre 104 y $205 \mathrm{mg} / \mathrm{L}$ que son iguales y hasta superiores a lo mostrado por las corontas iniciales. Esto refleja que tanto los procesos de fermentación como de conservación, logran afectar los contenidos en antocianinas, fenoles y flavonoides. Organolépticamente, la muestra $M$ - 3 fue la de mayor preferencia $(7,0$ puntos $=$ Bueno $)$ y catalogado como un vino semidulce de maíz morado.

Palabras clave : antocianinas, vino de maíz morado.

\begin{abstract}
The present investigation seeks to determine the possibility of transferring their wealth especially grains and purple corn cobs (Zea mays L), to transform it into an alcoholic beverage wine type.

We worked with four samples ( $M 1, M-2, M-3$ and $M-4$ ) that had the following composition: $50 \%$ malted grains, $10 \%$ unmalted grains and $40 \%$ of cobs. Once crushed, they were put to heat for 1 hour at a temperature of $75-85^{\circ} \mathrm{C}$ and water at four levels $(M-1=6.0 \mathrm{~L}, M-2=8.0 \mathrm{~L}, \mathrm{M}-3=100 \mathrm{~L}$ and $M-4=12.0 \mathrm{~L})$. Cooked musts increased by 3 to 5 times the initial value of the anthocyanins shown by dry cobs, corn regarding purple anthocyanin increases were between 7 and about 11 times due to the extraction process by leaching hot.

To facilitate the fermentation process of the four musts, tangy syrup added sucrose to raise their soluble solids from 5 to $22.5^{\circ}$ Brix, as well as $3 \%$ increase in the volume of white corn ferment foot Cuba.

They had four wines purple corn were stored and decanted four times, for 90 days and contained an alcohol content between $9^{\circ}$ and $10^{\circ} \mathrm{GL}$, pH between 3.1 and 3.3 as well as content in anthocyanins, between 104 and $205 \mathrm{mg} / \mathrm{L}$ which are equal and even better than that shown by the initial cobs. This reflects that both the fermentation process and storage, achieve affect the contents of anthocyanins, phenols and flavonoids. Organoleptically, the sample $M-3$ was the most preferred $(7.0$ points $=$ Good $)$ and listed as a semi-sweet wine made from purple corn.
\end{abstract}

Keywords: anthocyanin, purple corn wine.

'Ingeniero en Industrias Alimentarias, Magister en Ciencias con mención en Gestión Empresarial. Facultad de Ciencias Agropecuarias de la UNJBG. 


\section{INTRODUCCIÓN}

El propósito de la investigación fue rescatar e innovar las benéficas y funcionales bondades para la salud de las chichas espirituosas, elaboradas en base a las mazorcas de maíz morado (Zea mays L.), pero esta vez dándole un proceso de vinificación. El maíz morado se ha venido cultivando en los valles interandinos, desde hace más de 5000 años.

Los granos de maíz son fuentes importantes de carbohidratos, proteínas, minerales, vitaminas del complejo B, ácido ascórbico, todos ellos concentrados en el endospermo del grano. Además el maíz morado posee una rica composición en fitoquímicos, en especial antocianinas y compuestos fenólicos que tienen comprobados efectos funcionales en nuestro organismo.

Las antocianinas (unos seis grupos principalmente de cianin-3-glucosa o simplemente $\mathrm{C} 3 \mathrm{G}$ ) son pigmentos o colorantes flavonoides que dan la pigmentación natural azul, morada y roja en alimentos y bebidas. Estudios recientes les asignan importantes efectos benéficos como antioxidantes, anticancerígenos, estimulantes de la agudeza visual, reductor de riesgos cardio vasculares, regenerador del colágeno, etc.

Todos estos beneficios para la salud se desea incorporarlos en la elaboración de una bebida alcohólica tipo vino, con una graduación de alcohol entre 7 y $14{ }^{\circ}$ G.L. Su consumo puede considerarse importante en una región como Tacna que es vitivinicultora, pero también tiene una población muy expuesta a factores de riesgo contaminantes, entre ellos, la presencia de minerales pesados en el agua potable, en los recursos hidrobiológicos costeros, en la carne de ganado vacuno, caprino y porcino.

Son también negativos el exceso de plaguicidas en cultivos como frutales y olivo: el uso de aguas servidas para el riego de vegetales de tallo corto.

Los objetivos específicos del trabajo fueron:

a) Demostrar la relación maíz-agua en etapas de remojo y cocción.

b) Determinar parámetros en las etapas de remojo, malteado, cocción, fermentación y conservación.

c) Precisar las concentraciones de pigmentos de antocianinas presentes en la materia prima, es decir, en el agua de remojo, después de la cocción, en los orujos de la fermentación y en los vinos de maíz morado obtenidos.

d) Determinar las características físico-químicas y cualidades organolépticas de las bebidas alcohólicas obtenidas, tipo vino.

\section{MATERIALES Y MÉTODOS}

\subsection{Lugar de ejecución de la investigación}

El trabajo experimental de la investigación se desarrolló en las instalaciones y laboratorios de la E.A.P. de Ingeniería en Industrias Alimentarias, Facultad de Ciencias Agropecuarias de la UNJBG de Tacna.

\subsection{Materia prima, materiales, equipos $\mathrm{y}$ reactivos}

\subsubsection{Materia prima}

Se utilizaron mazorcas de maíz morado cuya procedencia, según los vendedores del mercado mayorista Grau, eran del "Valle de Tambo", Arequipa. No se encontró maíz morado de algún valle o irrigación de la región Tacna como era el propósito inicial.

\subsubsection{Materiales y equipos}

Los principales materiales y equipos para desarrollar este trabajo fueron:

Agua potable, alcoholímetro en \% vol., azúcar rubia /blanca, chicha de jora, balanza de platillo, balanza de precisión, baldes plásticos, bureta automática de titulación, calentador de baño maría, cápsulas de porcelana, cedazos y coladores, cocina a gas, cocinilla eléctrica, copas de vidrio para cata, densímetro de 1.1300 a $1.0000 \mathrm{~g} / \mathrm{L}$, depósitos de plástico y vidrio, estufa, espectrofotómetro, fichas de evaluación sensorial, kit de acidimetría, material de vidrio, molino de martillos para granos, ollas metálicas, pH-metro análogo, picnómetro, refractómetros de $\mathrm{O}$ a $35^{\circ} \mathrm{Brix}$, termómetros de $\mathrm{O}$ a $110^{\circ} \mathrm{C}$, utensilios de cocina. reactivos : ácido sulfúrico, ácido tartárico, hidróxido de sodio $0,1 \mathrm{~N}$, metabisulfito de potasio, reactivo de nelson (Sn2), reactivo de Somogy $(\mathrm{Sn} 1)$.

\section{RESULTADOS Y DISCUSIÓN}

\subsection{Materia prima}

\subsubsection{Humedad inicial}

Se determinó la humedad inicial por separado obteniéndose valores promedio de $14.08 \%$ para 
granos y $21.49 \%$ en las corontas. Alguna bibliografía indica $11.40 \mathrm{~g} / \mathrm{log}$, pero no especifica si es de granos o corontas.

\subsubsection{Contenido de las mazorcas}

El contenido porcentual promedio en las mazorcas fue de $81.62 \%$ en granos y $18.38 \%$ para las corontas.

\subsubsection{Composición química}

Al determinar la composición química de los granos se encontró lo siguiente:

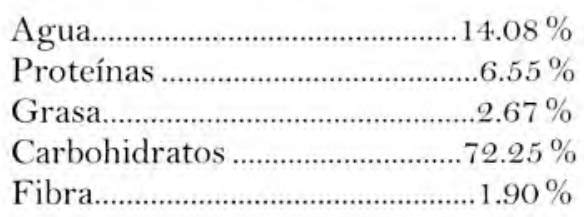

El tamaño de cada muestra fue de 1 kilogramo libre de materias extrañas.

\subsubsection{Contenido de antocianinas en la coronta y en granos}

Queda confirmado que los granos de maíz morado poseen menor contenido de compuestos antociánicos que las corontas, como se muestra en la Tabla $\mathrm{N}^{\circ} \mathrm{O} 1$.

La determinación se logró mediante el método espectrofotométrico específico propuesto por Giusti y Wrosltad (2001): "Extractos de antocianinas de coronta de Zea mays L morado" (citado por Arilmi Gorriti, 2009), por el cual se han medido dos valores de $\mathrm{pH}, 1$ y 4.5, dicha diferencial realizan las determinaciones, siempre con un mismo valor de dilución.

En los resultados se puede apreciar que el contenido de antocianinas en las corontas puede ser de 2.44 a 2.49 veces mayor que en los granos, según se exprese en $\mathrm{mg} / \mathrm{L}$ o en $\mathrm{mg} / \mathrm{loOg}$. Si queremos obtener mayor concentración de antocianinas debemos trabajar sólo con las corontas.

Tabla $\mathbf{N}^{\mathbf{0}}$ 01: Contenido de antocianinas en coronta y granos molidos de maíz morado.

\begin{tabular}{|c|c|c|}
\hline Muestra & $\begin{array}{c}\text { Antocianinas } \\
\text { (mg/L) }\end{array}$ & $\begin{array}{c}\text { Antocianinas } \\
\text { ( } \mathbf{m g} / \mathbf{1 0 0 g})\end{array}$ \\
\hline Coronta molida & 114.5660 & 916.5283 \\
Grano molido & 46.9770 & 367.8100 \\
\hline
\end{tabular}

Fuente: Propia

\subsection{Remojo de los granos}

Las muestras, conformaba $1 \mathrm{~kg}$ de granos, identificadas como M-1, M-2, M-3 y M-4, fueron puestas a remojar en 1.5 litros de agua potable, a temperatura ambiente, por un tiempo de 72 horas, con remoción dos veces al día, tras lo cual, la mayor parte de los granos se mostraban hinchados y aproximadamente el $35 \%$ tenían las raicillas de la germinación. Los granos fueron separados del agua que ya poseía una pigmentación morada.

Luego se determinó, por espectrofotometría, la presencia de antocianinas. Resultó eficaz el método espectrofotométrico específico propuesto por Giusti y Wrosltad: (2001) "Extractos de antocianinas de coronta de Zea mays L morado"(citado por Arilmi Gorriti, 2009), mediante el cual se realizan mediciones a 510 y 700 nanómetros, ambos a $\mathrm{pH}=1.0$ y $\mathrm{pH}=4.5$. La diferencia (delta) se presenta como resultado en la tabla siguiente.

Tabla No2: Contenido de antocianinas en agua de remojo de granos.

\begin{tabular}{|c|c|c|c|}
\hline Muestra & $\Delta \mathrm{pH}$ & Dilución & $\begin{array}{c}\text { Antocianinas } \\
(\mathrm{mg} / \mathrm{L})\end{array}$ \\
\hline 1 & 0.3784 & 11 & 68.4787 \\
2 & 0.3665 & 11 & 67.8726 \\
3 & 0.0502 & 11 & 92.6337 \\
4 & 0.3803 & 11 & 70.5544 \\
\hline
\end{tabular}

Fuente: Propia

\subsection{Germinación o malteado de los granos}

Esta etapa del proceso genera una serie de cambios bioquímicos en el interior de los granos, proceso conocido como "amilólisis" o degradación de las moléculas de almidones por las enzimas $\alpha$ amilasa, $\beta$-amilasa, proteasas, citasas y otras. Además influyen en el proceso de fermentación de los azúcares.

\subsection{Oreado y tostado}

Los granos germinados se mostraban húmedos por lo que se dejó orear al medio ambiente por 48 horas, luego cada muestra fue sometida al proceso de tostado por calor seco, a temperaturas entre 70 y $80^{\circ} \mathrm{C}$ por 20 minutos.

Ese rango de temperaturas y tiempo se determinó con el fín de no afectar significativamente la calidad de los pigmentos de antocianinas. 


\subsection{Proporción de componentes y tamaño de las muestras}

Es conveniente establecer una adecuada proporción en las muestras de granos malteados y sin maltear, así como de las corontas para que juntos provean de pigmentos, azúcares fermentecibles y enzimas catalizadoras.

La proporción es como sigue : Granos malteados $50 \%$, granos no malteados $10 \%$, corontas $40 \%$.

\subsection{Proporción de la dilución sólido-líquido}

Para tener suficiente componente líquido en la cocción y fermentación, es necesario que cada muestra de maíz tenga diferentes proporciones de agua.

En nuestro caso quedó establecida la siguiente proporción sólido - líquido (diluciones): ${ }^{*} \mathrm{M}-1=1$ $\mathrm{kg}: \quad 6 \mathrm{~L}$ de agua. ${ }^{*} \mathrm{M}-2=1 \mathrm{~kg}: 8 \mathrm{~L}$ de agua. $* \mathrm{M}-$ $3=1 \mathrm{~kg}: 10 \mathrm{~L}$ de agua. ${ }^{*} \mathrm{M}-4=1 \mathrm{~kg}: 12 \mathrm{~L}$ de agua.

\subsection{Cocción}

Las cuatro muestras fueron cocidas por separado, con la adición de pequeñas cantidades de saborizantes naturales, entre ellos, trozos de piña fresca, canela, clavo de olor. Los parámetros fueron: temperatura en un rango de $75-85^{\circ} \mathrm{C}$ y tiempo de una hora para no afectar los pigmentos de antocianinas y fenoles.

\subsubsection{Características físico-químicas de los mostos cocidos}

Tabla $\mathbf{N}^{\circ}$ 03: Características físico -químicas de los mostos cocidos.

\begin{tabular}{|c|c|c|c|c|c|c|}
\hline Muestra & $\begin{array}{l}\text { Volumer } \\
\text { (L) }\end{array}$ & $\begin{array}{c}\text { Densidad } \\
(\mathrm{g} / \mathrm{L})\end{array}$ & $\underset{\left({ }^{\circ} \mathbf{B x}\right)}{\text { S.S. }}$ & \multicolumn{2}{|c|}{$\begin{array}{l}\text { Ac. Total } \\
\text { Sulf. Acet. }\end{array}$} & $\mathrm{pH}$ \\
\hline M & 3.5 & 1. & 6.0 & 2 & 3. & 9 \\
\hline $\mathrm{N}$ & 4. & & 3.5 & & & .9 \\
\hline & & & 2.5 & & o. & 5.5 \\
\hline M & 9.2 & 1.010 & 2.0 & 0. & 0.6 & 5.5 \\
\hline
\end{tabular}

Fuente: Propia

Como se ve, la cantidad de orujos obtenidos en cada muestra fueron: $* \mathrm{M}-1=2.52 \mathrm{~kg},{ }^{*} \mathrm{M}-2=2.67$ $\mathrm{kg},{ }^{*} \mathrm{M}-3=2.56 \mathrm{~kg},{ }^{*} \mathrm{M}-4=2.59 \mathrm{~kg}$. La humedad residual alcanza el promedio de $17.5536 \%$.

\subsubsection{Contenido de antocianinas en los mostos cocidos}

El contenido de antocianinas en mostos cocidos según sus disoluciones se muestra en la siguiente representación:

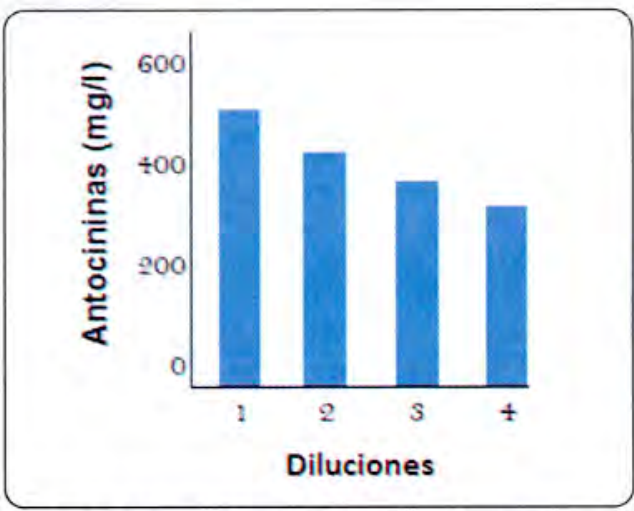

Gráfico $\mathrm{N}^{\mathbf{0}}$ 01: Contenido de antocianinas en mostos cocidos según sus diluciones.

\subsection{Adición de jarabe acidulado de sacarosa}

De acuerdo a la Tabla $\mathrm{N}^{\circ} 03$ vemos que los sólidos totales no son los suficientes como para asegurar un proceso fermentativo alcohólico. Por eso se agregó un jarabe acidulado de sacarosa de $62.5^{\circ}$ Brix en la cantidad suficiente para que todos los mostos iniciaran la fermentación con $22.5^{\circ}$ Brix iniciales.

\subsection{Adición de pie de cuba o fermento}

Se agregó a cada muestra ( $3 \%$ de volumen en promedio) un fermento de maíz blanco de ocho días de proceso, conteniendo levaduras alcohólicas. Este fermento tuvo una densidad de $1.044 \mathrm{~g} / \mathrm{L} \mathrm{y}$ $\mathrm{pH}$ de 3.5 medidos a $20^{\circ} \mathrm{C}$.

\subsection{Adición de conservante selectivo}

A cada muestra se agregó metabisulfito de potasio en solución, equivalente a $10 \mathrm{~g} / \mathrm{Hl}$.

\subsection{Control de fermentación de mostos}

Tabla $\mathbf{N}^{\circ}$ 04: Control de parámetros al término de la fermentación.

\begin{tabular}{|c|c|c|c|c|c|c|}
\hline Muestra & $\begin{array}{c}\text { Volumen } \\
(\mathbf{L})\end{array}$ & $\begin{array}{c}\mathbf{T}^{\mathbf{o}} \\
\left({ }^{\circ} \mathbf{C}\right)\end{array}$ & $\begin{array}{c}\text { Densidad } \\
(\mathbf{g} / \mathbf{L})\end{array}$ & $\begin{array}{c}\text { S.S. } \\
\left({ }^{\circ} \mathbf{B x}\right)\end{array}$ & $\mathbf{p H}$ & $\begin{array}{c}\text { Ac. } \\
\text { Total } \\
(\% \text { Sulf. })\end{array}$ \\
\hline M1 & 3.0 & 17 & 1.017 & 10.5 & 4.9 & 3.67 \\
M2 & 5.5 & 17 & 1.025 & 11.5 & 4.9 & 4.46 \\
M3 & 7.0 & 17 & 1.027 & 12.5 & 5.5 & 3.82 \\
M4 & 9.0 & 19 & 1.026 & 12.0 & 5.5 & 3.96 \\
\hline
\end{tabular}

Fuente: Propia

\subsection{Controles en los vinos terminados}

Después del último trasiego, los vinos de maíz morado fueron depositados en envases de vidrio, luego se obtuvieron muestras para realizar los controles físicos, químicos, de antocianinas y de aceptación organoléptica. 
Tabla $\mathrm{N}^{\circ} \mathbf{0}$ 5: Contenido de antocianinas en vinos de maíz morado.

\begin{tabular}{|c|c|}
\hline $\begin{array}{c}\text { Muestra según } \\
\text { Dilución }\end{array}$ & $\begin{array}{c}\text { Antocianinas } \\
(\mathbf{m g} / \mathrm{L})\end{array}$ \\
\hline M1 - (1:6) & 205.3 \\
M2 - (1:8) & 142.5 \\
M3 - (1:10) & 106.3 \\
M4 - (1:12) & 104.7 \\
\hline
\end{tabular}

Fuente: Propia

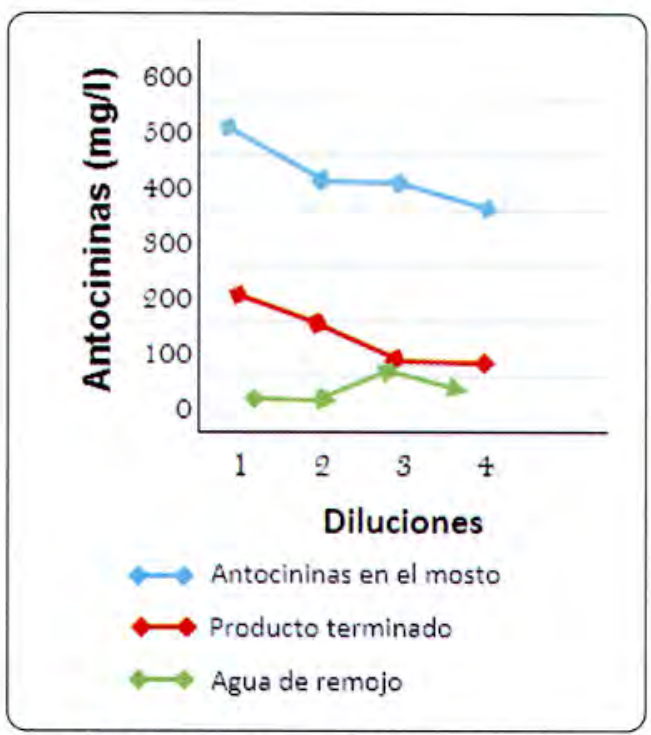

Gráfico $\mathrm{N}^{\circ}$ 02: Contenido de antocianinas en tres etapas del proceso.

\subsection{Evaluación organoléptica hedónica de los vinos de maíz morado.}

Tabla $N^{\circ}$ 06: Muestras obtenidas luego de la evaluación organoléptica

\begin{tabular}{|l|c|}
\hline Muestras & $\begin{array}{c}\text { Puntuaje } \\
\text { promedio }\end{array}$ \\
\hline M1 (627) & 6.79 \\
M2 (896) & 6.25 \\
M3 (1111) & 7.00 \\
M4 (1253) & 6.50 \\
\hline
\end{tabular}

\section{Fuente: Propia}

La muestra M-3 resultó la mejor, pues sumó 7.0 puntos y se califica hedónicamente como buena. Se denota como el vino más equilibrado. Le sigue en preferencia la muestra $\mathrm{M}-1$ que alcanzó 6.79 puntos, y también se la puede considerar buena. Muestra el color morado más intenso. Se caracteriza por tener el menor contenido en dulzor. La muestra $\mathrm{M}-4$ fue calificada con 6.50 puntos y tipificada como regular, al percibir un ligero olor que recuerda a levaduras del proceso fermentativo. Finalmente la muestra $\mathrm{M}-2$ fue la que menor puntaje obtuvo, 6.25, considerándose como un vino regular debido a un exceso de acidez y un gusto, un tanto anómalo, probablemente originado en la etapa de fermentación complementaria.

\section{CONCLUSIONES}

- Se aprecia una diferencia significativa en el contenido de antocianinas encontradas en las corontas (114.56 mg/L), respecto de los granos de maíz morado $(45.97 \mathrm{mg} / \mathrm{L})$.

- En el agua de remojo de granos (72 h a temperatura ambiente), se encontraron significativas cantidades de antocianinas que varían entre 67.87 y $92.63 \mathrm{mg} / \mathrm{L}$ ).

- La proporción granos/coronta, que fue determinada inicialmente en $60 / 40$, no habría sido la más adecuada para obtener una mayor extracción de pigmentos de antocianinas y fenoles en los mostos y vinos, pero sí, para favorecer el proceso fermentativo debido a los almidones y azúcares fermentecibles presentes en los granos malteados.

- El proceso de cocción de las cuatro muestras, $\left(1 \mathrm{~h}\right.$ a $\left.75-85^{\circ} \mathrm{C}\right)$, que tuvieron diluciones crecientes ( $1 / 6,1 / 8,1 / 10$ y $1 / 12$ litros), aún cuando no llegaron a temperaturas de ebullición, significó un incremento en la extracción y contenido de antocianinas en orden decreciente de acuerdo a cada dilución.

- Los mostos cocidos y fríos, antes de la fermentación, mostraron contenidos de antocianinas, entre 3 a 5 veces mayores que la materia prima coronta y entre 7 a 11 veces mayor, respecto de los granos de maíz morado.

- Las bebidas finales, vinos de maíz morado, muestran significativas pérdidas en sus valores de antocianinas residuales al compararlos con los valores de los mostos cocidos, como consecuencia de su fermentación y posterior conservación. La muestra $\mathrm{M}-1$ perdió $59.12 \%$; la $\mathrm{M}-2,63.5 \%$; la $\mathrm{M}-3,71.7 \%$; y la $\mathrm{M}-4$, perdió $69 \%$. A mayor dilución mayores fueron las pérdidas.

- Organolépticamente, la mayor preferencia la obtuvo la muestra $\mathrm{M}-3$ (7.0 puntos), seguida de la $\mathrm{M}-1$ (6.79); la $\mathrm{M}-4(6.5)$; y finalmente la $\mathrm{M}-2$ (6.25). 


\section{REFERENCIAS BIBLIOGRÁFICAS}

Bush, Carlos (1952). Consideraciones médicas sociales sobre la chicha. Periódico Excelsior $\mathrm{N}^{\circ}$ 127-mayo-junio. Lima, Perú. Páginas 25-27.

Collazos, Carlos et al. (1975). La composición de los alimentos peruanos. MINSA-INS-INN. Quinta edición, Lima, Perú. Páginas 1-19

Cerro Q., Romina (2009). Extraçao e Caracterizaçao do Pigmento do Sabugo de Mihlo Roxo (Zea mays L.) e sua Aplicaçao em Alimentos. Título de Mestre em Ciencia de Alimentos. Faculdade de Engenharia de Alimentos. Universidad Estadual de Campinas. Sao Paulo, Brasil.

Garzón, Gloria. Ph.D. (2008).Las antocianinas como colorantes naturales y compuestos bioactivos: Revisión. Acta Biológica Colombiana. Vol $13, \mathrm{~N}^{\mathrm{o}} 3,2008$. Pag.27-36.

Gonzalez-Manzano, Susana., et al (2008).Flavonol-anthocyanin pigments in corn:NMR Characterisation and presence in different purple corn varieties. Jornal of Food Composition and Análysis.Vol 21.(2008).Pag. 521-526.

Kuskoski,E. Marta, et al. (2004). Actividad antioxidante de pigmentos antociánicos. Ciencia e Tecnologia de Alimentos. Vol 24, $\mathrm{N}^{\circ}$ 4.Campinasout./ dez 2004.Pag. 1-7.
Lock Sing de Ugaz, Olga (1997).Colorantes naturales. Pontificia Universidad Católica del Perú. Fondo Editorial. Lima, Perú. Páginas 1-9; 95-116;205-218.

Manrique de Saenz, Isabel (1978). Flora Microbiana de la Chicha de Jora y Fermentación Experimental de Levadura Seleccionada. Tesis de Grado. programa de Farmacia. UNMSM. Lima, Perú.

Muelle, Jorge (1945). La Chicha en el distrito de San Sebastián, Cusco. Revista del Museo Nacional. Tomo XIV. Páginas 114-124 9. Portocarrero C, Miguel Angel. (2012). Tabla de Composición Química de Alimentos de Mayor Consumo en el Perú. Sexta edición, 1993. Lima, Perú.

Gorriti G.,Arilmi, Quispe J.,Jorge, Arroyo A., Jorge et al. (2009). Revista Ciencia e Investigación: Extracción de Antocianinas de las corontas de Zea mays L. Maíz Morado. Facultad de Farrmacia y Bioquímica. UNMSM - Unidad de I.D.I. Empresa Agronegocios PeruAgro SRL. Pags 64-74. Lima, Perú.

URL: http://www.alambiques.com/ alambiques. html

Correspondencia:

Samuel Román Cerro Ruiz

Ciudad Universitaria fundo "Los Granados"

Av. Miraflores s/n Tacna - Perú

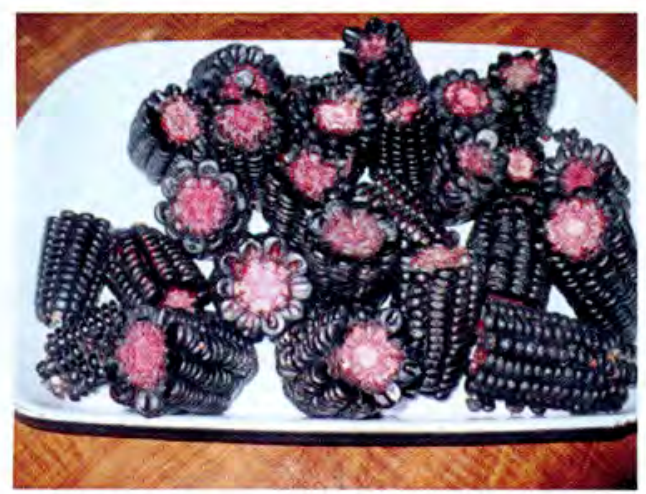

Muestra de mazorcas de maíz morado

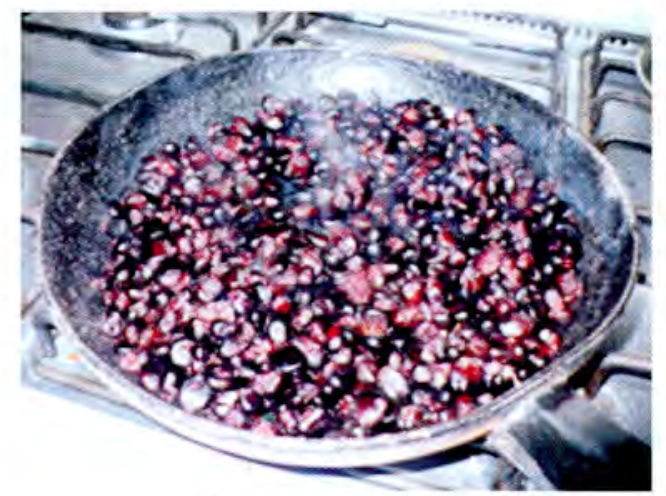

Tostado de granos 


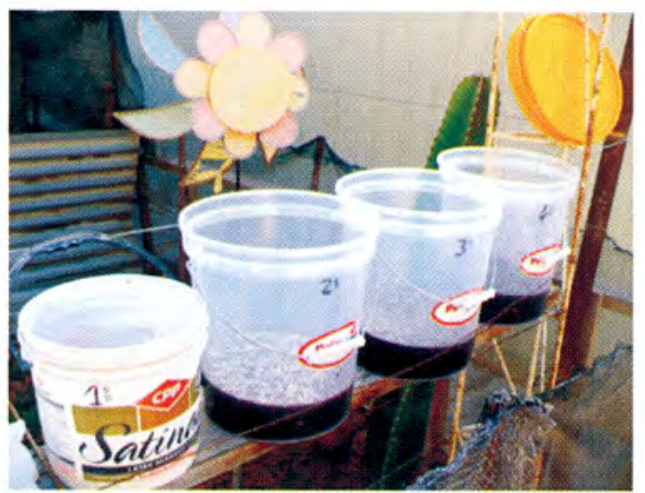

Muestras de granos en remojo

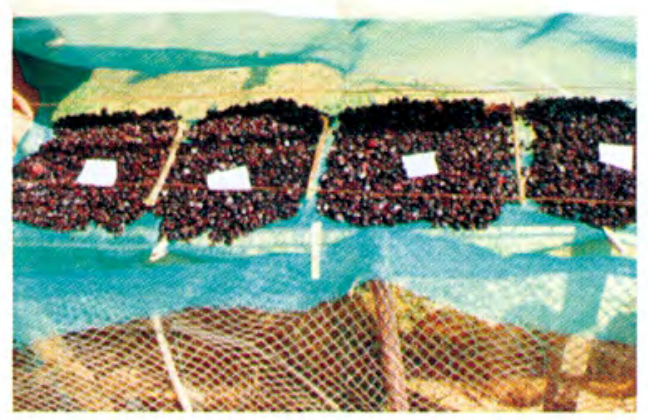

Proceso de malteado de granos de maíz morado

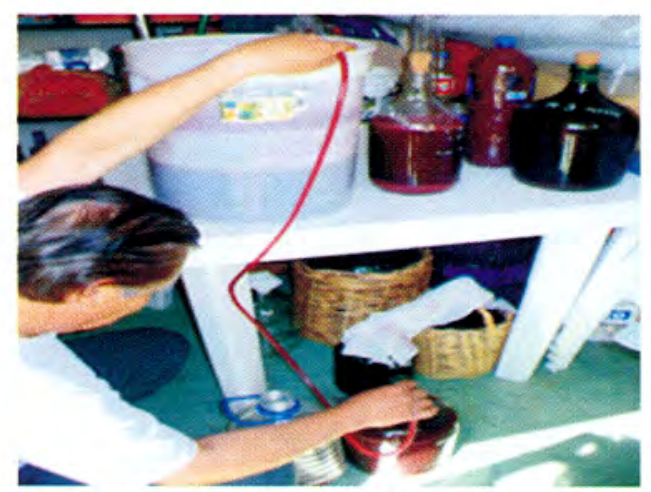

Trasiego de vinos de maíz.

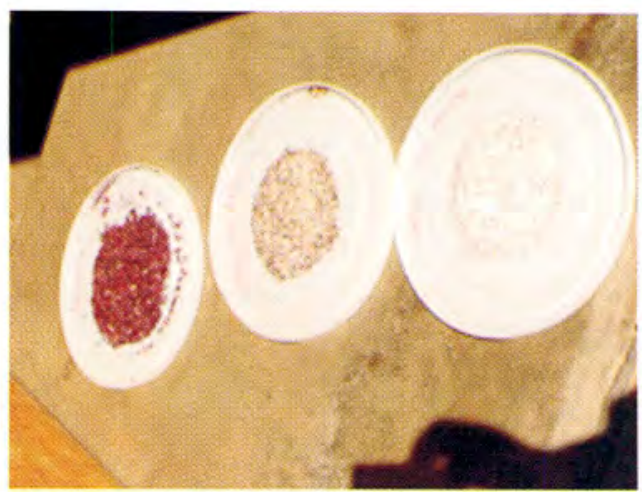

Muestra de coronta y granos molidos

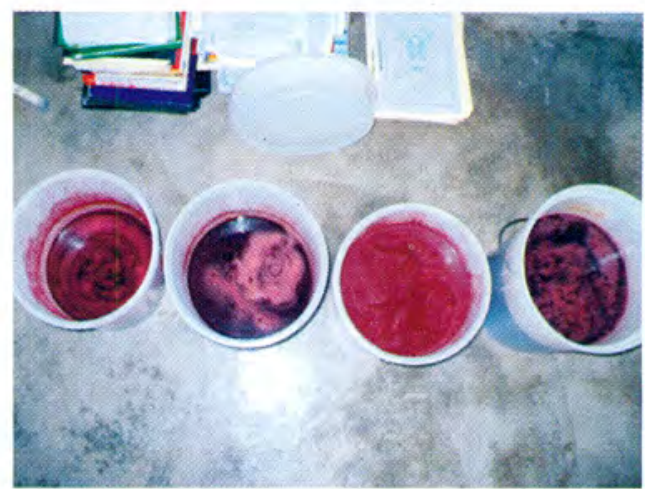

Fermentación de las cuatro muestras.

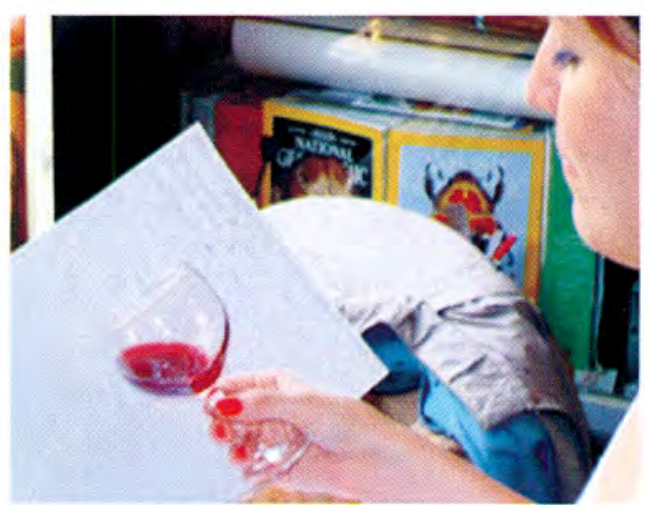

Evaluación organoléptica de los vinos. 